\title{
06 ADVANCED CARE PLANNING FOR PEOPLE WITH INTELLECTUAL AND DEVELOPMENTAL DISABILITIES
}

T. A. Savage ${ }^{1}$, T. Moro', J. Boyden', A. Brown ${ }^{2}$ and K. Kavanaugh ${ }^{1}$ University of Illinois at Chicago College of Nursing, Chicago, IL; ${ }^{2}$ Hines VA Hospital /Jesse Brown VAMC

10.1136/bmjspcare-2012-000250.105

Over 4 million people in the U.S. have an intellectual or developmental disability (I/DD). While most people with I/DD have an equivalent life expectancy to that of the general population they experience numerous barriers to quality health care at the end of life. End-of-life care, including advanced care planning, is not well understood for individuals with I/ $\mathrm{DD}$. In order to elucidate end-of-life care for people with I/ $\mathrm{DD}$, a qualitative, exploratory approach was used to collect focus group and individual interview data from individuals with I/DD and their family and staff caregivers. Participants were recruited from four community agencies. While people with I/DD were able to discuss their experiences when they lost someone close to them, it proved challenging to describe concrete issues such as advanced care planning and medical care at the end of life. The family members' and staff vividly recalled their experiences when their loved one was at the end of life. The quality of care received was variable. Some participants described compassionate care, while others perceived a poor quality of care. In general, it was reported that individuals with I/DD were not involved in advanced care planning and rarely had formal plans in place. The aim of this paper is to present end-of-life care for people with I/DD as perceived by their family and staff caregivers and to discuss the barriers to end-of-life care and advanced care planning for people with I/DD. Recommendations for including people with I/DD in their end of life care will be discussed. 